\title{
Cronos: A Separate Compilation Toolset for Modular Esterel Applications
}

\author{
Olivier Hainque ${ }^{1 \star}$, Laurent Pautet ${ }^{1}$, Yann Le Biannic ${ }^{2}$, and Éric Nassor ${ }^{2}$ \\ 1 École Nationale Supérieure des Télécommunications \\ 46, rue Barrault - F-75013, Paris - France \\ hainque@inf.enst.fr - pautet@inf.enst.fr \\ 2 Dassault-Aviation \\ 78, quai Marcel Dassault - F-92214, Saint-Cloud Cedex - France \\ eric.nassor@dassault-aviation.fr - yann.lebiannic@dassault-aviation.fr
}

\begin{abstract}
ESTEREL is an imperative synchronous language designed for the specification and the development of reactive systems. Recent studies pointed out that its use for the development of avionics software can yield great benefits but that the lack of support for separate compilation in the current toolset may be an obstacle to the development of large systems. This paper presents the CRONOS framework which provides such support for some specific cases of ESTEREL programs.
\end{abstract}

Technical Paper related to Industrial Applications

\section{Keywords :}

Esterel, Compilation, Synchronous Reactive Systems, Avionics Software

\section{Introduction}

Dassault-Aviation is a French aircraft manufacturer which has been studying the industrial applicability of the ESTEREL synchronous language in the development process of avionics software since 1989. Some recent experiments [4], [5] showed that using ESTEREL in this context can actually yield great benefits but that the current toolset is missing some separate compilation capabilities to be applicable to large industrial systems. This paper presents the CRONOs framework developed by Dassault-Aviation to deal with this issue for some specific cases of ESTEREL programs.

Sections 2 and 3 provide a short background about ESTEREL and a basic example which will be used as an illustrative support for the following sections. Section 4 describes the current compilation process and points out its major weaknesses regarding its applicability to large systems. Sections [5 and [6] explains how the CRONOs toolset balances these weaknesses and details the kind of applications it can handle. Finally, sections 7 and 8 summarize the major advantages of the approach and present the results of experiments conducted to evaluate it.

* Contact author : O. Hainque - Tel : (+33) 1471136 94, Fax : (+33) 147115283

J. Wing, J. Woodcock, J. Davies (Eds.): FM'99, Vol. II, LNCS 1709, pp. 1836-1853 1999.

(C) Springer-Verlag Berlin Heidelberg 1999 


\section{Basic Esterel Background}

ESTEREL is an imperative synchronous language designed for the specification and the development of reactive systems [1].

A reactive system is an entity which produces output signals and updates its internal state when triggered by input events coming from its environment. The environment generates the events at its own rate and each handling of an event by the system is called a reaction.

In this context, the synchronous approach first makes the assumption that the reaction execution time is null and defines a discrete logical time scale from instants corresponding to each reaction (Fig.11). Reactions are then said to be instantaneous and all the associated triggering inputs can be considered as strictly simultaneous. This strict characterization of the notions of instantaneity and simultaneity allows the definition of very precise semantics from which highly deterministic behaviors can be derived. Naturally, the actual execution time of a reaction is never null, but in practice the results are applicable as soon as it is possible to ensure that a reaction is always complete before the occurrence of the next event.

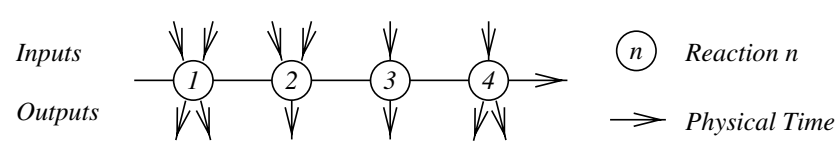

Fig. 1. Synchronous Reactive Systems : a discrete logical time scale

ESTEREL allows the description of a synchronous system as a set of interconnected modules. Inter-modules and system-environment communications are achieved through signal based interfaces, with pure signals simply indicating individual events and valued signals able to carry additional data. The modules behavior is specified thanks to a rich set of imperative constructs, including support for exceptions, preemptions and parallelism. The full description of the language and of its semantics is beyond the scope of this article but can be found in [2], [3], and in the documentation distributed with the compiler.

\section{A Simple Example}

As an illustrative support for the following sections, we shall consider the case of a simple system called COMCTL with a controller CTL and a controlled component COM (Fig.2). At the environment level, a Switch signal alternatively activates or deactivates the component and a Status_Rq signal requests the controller to emit a COM_Is_Active or COM_Is_Inactive indication corresponding to the current component state. 
We have specified this with three modules : one for COM, one for CTL and one to combine them and build the system using a couple of internal signals, Activity_Rq and Activity. Every time CTL receives Status_Rq, it emits Activity_Rq to which COM instantaneously replies by an Activity indication if the component is currently active. CTL then tests the presence of this indication to emit one of the two expected system outputs.

With run, ; and II as respectively the module instantiation, sequencing and parallelism operators, we obtain the organization and code on Fig.2 :

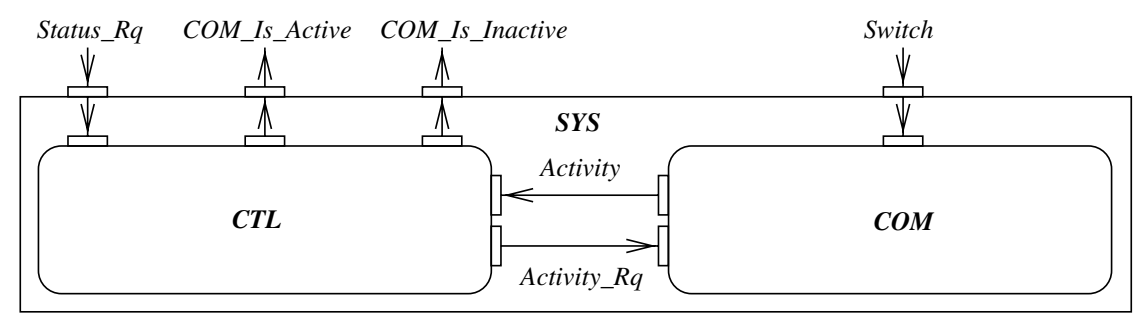

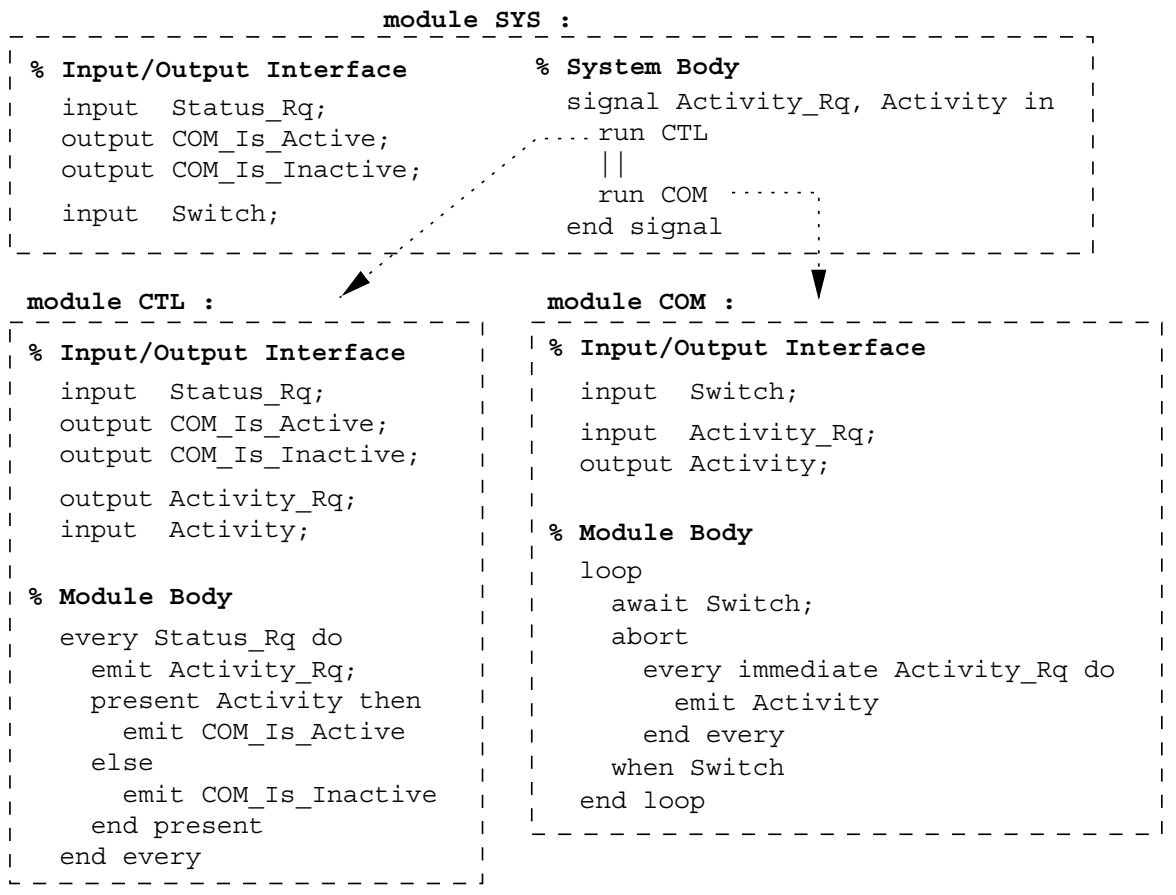

Fig. 2. General organization of $\mathrm{COMCTL}$

Fig. 3] shows an example sequence of reactions of this system, each represented by a circle with the triggering inputs above and the emitted outputs below. 


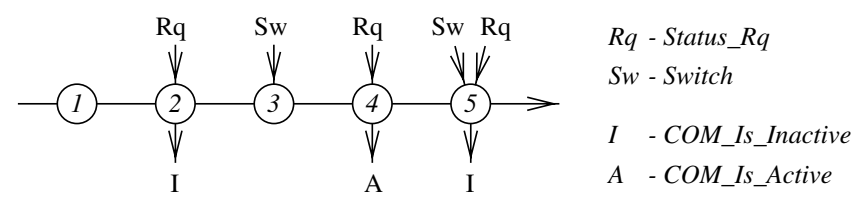

Fig. 3. An example sequence of reactions for COMCTL

\section{The Esterel Compilation Process}

\subsection{General Description}

From a given specification, the latest V5 release of the ESTEREL compiler generates a boolean sequential circuit equivalent to a finite state machine for the control aspects. The system inputs/outputs are connected to input/output wires and a set of boolean latches encodes the state between two consecutive reactions (Fig.4). Data handling, such as if statements or variable assignments, is operated outside the circuit by operations called actions, triggered by wires evaluated to true and possibly returning information back into the circuit through another wire. The execution of a reaction basically consists in computing the status (emitted or not) of every output and the next system state from the provided status (present or not) of all the inputs combined with the current state. In this context, every signal which status is known (provided or computed) is said to be determined.

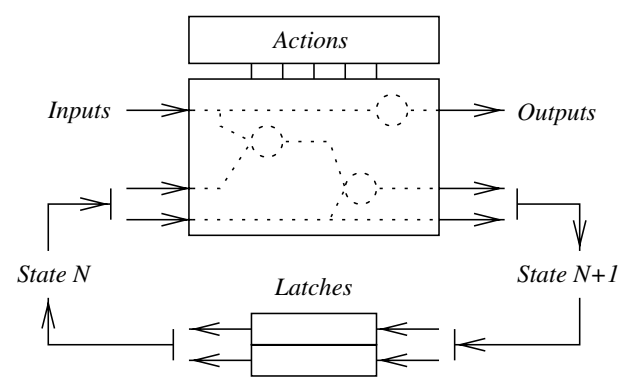

Fig. 4. General form of a circuit generated by ESTEREL

This approach has two interesting advantages :

- The logical part of the circuit can be submitted to very efficient specialized optimization tools,

- The state encoding enables to handle pretty huge state spaces (up to $10^{14}$ states reached in Dassault-Aviation applications). 
If we consider the CTL module as an independent system and submit it to the EsTEREL toolset, we get an example on Fig. 5 :

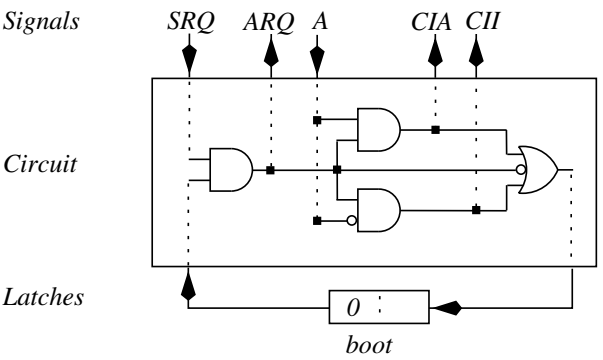

SRQ: Status_Rq present?

A : Activity Present?

ARQ: Emit Activity_Rq

CII : Emit COM_Is_Active

CIA : Emit COM_Is_Inactive

Fig. 5. Circuit translation for CTL

The corresponding generated $\mathrm{C}$ code includes one function for each input signal and one function to request a reaction. This function executes considering as present only the input signals which associated function has been called at least once since the previous reaction. It evaluates the gates in topological order, calls a user provided function for every emitted output, updates the registers and resets the inputs status to false (absent) before returning.

The boot register visible on the figure has a 0 initial value and is subsequently always set to 1 . As its name suggests, it provides an indication of whether or not we are running the very first reaction. It is there due to a specificity of the EsTEREL every construct which starts looking at the condition only one instant after its own activation, unless the condition is prepended by immediate. Thus, our CTL module actually never emits anything at the first instant, even if Status_Rq is present, as ensured by the leftmost and gate in the circuit layout. This is not a serious problem and provides a nice simple example of the use of registers to encode the system state.

A very important point about this general scheme is that a reaction always leads to the evaluation of all the gates. This approach significantly differs from the one adopted for other synchronous languages such as Lustre or SignaL which only evaluate parts of an overall dataflow graph depending on the status the system inputs ([12], [11], [10]).

\subsection{Major Translation Steps}

The ESTEREL compilation environment achieves the translation of a specification through various intermediate tools and formats. It is not possible to precisely describe them in this article, but some key points are important to understand what the issues addressed by CRONOs are and how the solutions will take advantage of the existing toolset. 
Fig. [6] below shows the most important steps for our example and purpose of presentation :

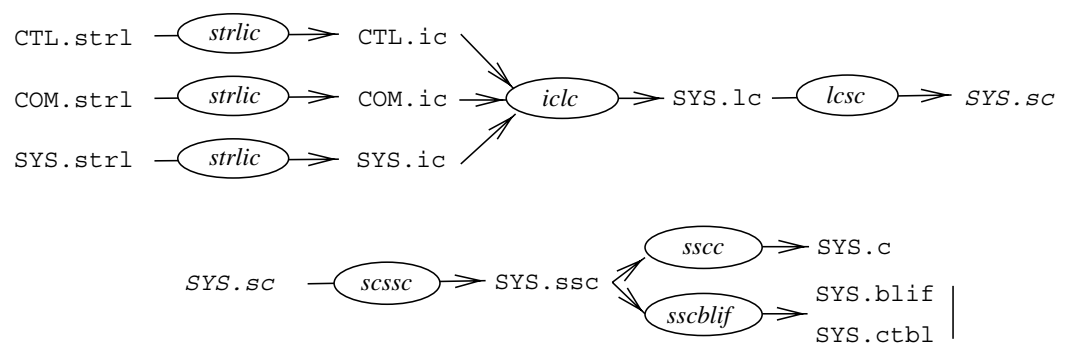

Fig. 6. Esterel to circuit translation for COMCTL

The first format (ic for Intermediate Code) enables the internal representation of all the necessary modules. The resulting files are linked together by iclc to build a 1c (Linked Code) representation of the entire system. 1csc then transforms this representation into a boolean equation system associated with a set of tables describing the actions and their relations with the equations (Software Code). A topological sort of the equations by scssc yields a ssc file (Sorted sc), from which sscc can directly produce a $\mathrm{C}$ emulation of the circuit.

Starting from a ssc file, it is also possible to get a pure description of the circuit in the standard blif format (Berkeley Logic Interchange Format) associated with a ctbl file (Common Tables) for the tables. blif level optimizers are then usable together with a blifssc processor to build a ssc file from which a much more efficient $\mathrm{C}$ code can be obtained.

\subsection{Weaknesses When Applied to Large Systems}

As illustrated by Fig. 6 a representation of the entire system is built very early during the translation process and is then submitted to a significant number of operations. An immediate consequence is that any modification to any module imposes to apply again almost the whole translation process to the entire system, which is not reasonable when hundreds or thousands of modules are concerned.

Moreover, some compilation steps sometimes require the execution of costly algorithms which should only be applied to a specific part of the system. For instance, if the combinational part of the circuit contains cycles despite a semantically correct specification, producing the ssc file requires the use of a causality analyzer to compute an equivalent acyclic circuit (SCCAUSAL : 8], 9]). The involved analysis is complex and performed at the sc level, that is for the whole system, even if only one specific module is the source of the anomaly.

Finally, the optimization processes we have mentioned are often necessary to reach acceptable reaction execution times, but are sometimes computationally not tractable when the system is too large. 


\section{Separate Compilation with Cronos}

\subsection{Basic Assumptions and Ideas}

CRONos basically consists in a set of code generation tools for ESTEREL which enable to delay the modules assembly step until the very end the overall compilation process. To achieve this goal at a reasonable cost, we first consider the following restriction :

The root level description of the system only contains parallel module instantiations with possible local signals for their interconnection. 1

This allows to consider the whole system circuit as a simple signal-level interconnection of the circuits for each module and to bind the separate compilation issue to a two steps process :

1. Generating the code for each module from the representation of its circuit which is trivially obtainable with the existing ESTEREL tools;

2. Generating the code for the interconnection of these circuits as it is specified by the root level description of the system.

This may seem pretty simple at first sight but the possible occurrence of instantaneous bidirectional communications between modules raises a critical problem. As described in section 4.1, a full reaction of any module corresponds to the evaluation of the whole associated circuit and requires all the module inputs to be determined. Thus, instantaneous bidirectional communications prevent to simply build a global system reaction from a sequence of module reactions because no execution order satisfies all the signal determination precedence constraints. In the CoMCTL case, for instance, the Activity/Activity_Rq loop would require each module to react before the other (CTL before COM to determine Activity_Rq and COM before CTL to determine Activity).

This problem has already been mentioned in [13] which proposed to reject the interconnection if it does not include at least one logical delay to break the instantaneity of the communications. We observed through several experiments that this solution imposes serious restrictions upon applications structure. Indeed, true causality problems come from instantaneous cyclic dependencies between signals, which are not implied by such dependencies between modules. An alternative is to build a consistent system reaction from an ordered sequence of partial evaluations of each module circuit, which is possible as soon as the combinational part of the overall system circuit is acyclic. This requires a deep analysis of the module circuits and a careful integration but greatly relaxes the final constraints. We decided to support this in CRONOs with a couple of tools implementing the generation steps mentioned above and presented in the following sections. The first one is called mgen and generates the code enabling the part by part evaluation of one module circuit as its inputs get determined. The second one is called mbind and generates the code for a specific composition of parallel modules previously submitted to mgen. It also checks for the overall acyclicity constraint that will be further discussed.

\footnotetext{
${ }^{1}$ We will now denote by "modules" the modules instantiated at the root level.
} 


\subsection{Individual Modules Analysis}

This step consists in generating for each module the code that enables to evaluate its associated circuit incrementally as its inputs get determined. Such a generation is the result of an analysis explained in this section and from which is also produced an information file required to allow the further integration of the modules into a system parallel composition.

The natural starting point for the generation of a circuit evaluation is the description of the circuit itself, that is the blif/ctbl representation of the module which can easily be obtained with the existing EsTEREL tools. The blif file contains the description of the logical part of the circuit, including the boolean registers. The ctbl file contains the description of the actions, of their connections with the circuit wires, and of some ordering constraints which have to be enforced but which were only implicit at the ssc level. mgen analyzes them and builds an internal representation of the circuit as a partially ordered graph of evaluation nodes for gates and actions, with arcs expressing precedence constraints. Fig.7] below provides an example of such graph for the CTL module.

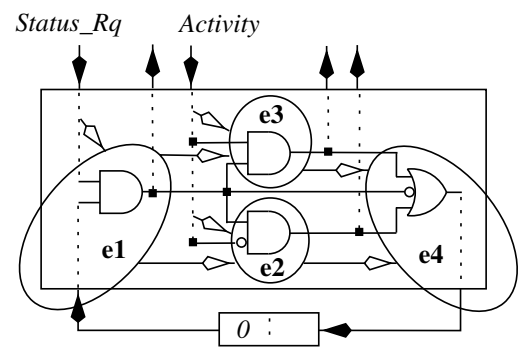

Fig. 7. Cronos evaluation splitting for the CTL module

The figure shows four evaluation nodes for CTL, symbolized by the e1 to e4 ovals. e1 and e4 are a bit larger because they respectively perform latch reading and latch update operations. The arrows inside the circuit box represent the precedence constraints which determine the graph partial order. Arrows which do not have their origin on a node but on an input wire indicate a precedence constraint between the determination of this input and the possibility to evaluate the node to which the arrow points.

In this specific case, e1 can be evaluated as soon as Status_Rq is determined, but not before because the gate it computes uses the corresponding wire. e2 and e3 can only be evaluated once Activity is determined and after e1 for similar reasons. Finally, because the rightmost or gate uses the wires computed by all the others, the node which computes it shall only be evaluated after all the others. The e1 to e 4 precedence constraint is not on the figure and is actually removed by mgen since it is trivially enforced by transitivity. The latches update corresponds to an actual state transition and is always performed after everything else. 
The context into which a module will be instantiated is not known a-priori. The order of its inputs determination is then undefined and some triggering mechanism is required to schedule the nodes execution at run-time. This becomes costly as the graph gets larger and it is clear that one node for each gate and action will easily yield a huge number of nodes. To deal with this, mgen executes a graph compression algorithm which preserves all the dependencies as well as the possibility to evaluate parts of the circuit progressively with input determinations. In the CTL example, e2, e3 and e4 can be safely merged into an evaluation group eg1 linked after e1 and the determination of Activity. This does not change the basic principles regarding the separate compilation issue but helps a lot in getting an industrially usable result in terms of memory footprints and efficiency. For illustration purposes, we will keep considering the CTL graph before the compression step because it is clearly closer to the structure of the underlying circuit.

mgen first produces code which reflects this structure. Its detailed architecture is not useful at this point of the paper and is presented in 5.4

It also generates a textual representation of the module evaluation graph and of all the related dependencies, as summarized by Fig.8 for CTL. This description additionally includes what we call output supports, which express for each output signal of the module the inputs on which it depends via some path in the graph.

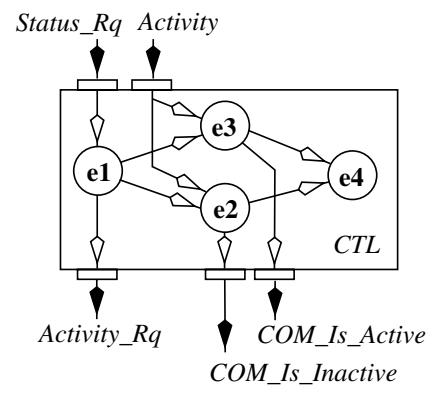

Fig. 8. CRonos evaluation graph for the CTL module

All these information will be the base of the mbind processing to integrate instances of the module into an outer parallel composition, as explained in the following section.

One important aspect is that we actually achieve true separate compilation since the code for each module is produced without any information about the context into which it will be instantiated. This prevents from performing some optimizations but allows to use the generated binaries in different contexts, which is crucial when such binaries are involved in certification processes. 


\subsection{Binding the Modules Together}

After the analysis of each module by mgen, the Cronos mbind tool enables to bind them together accordingly with a purely parallel composition specification. Since this specification is also an ESTEREL module, the basic goal is to generate code with the same interface as the one produced by mgen and possibly integrable into a still outer purely parallel system.

The starting point is the ESTEREL source file of the composition module, which specifies the interconnection of all the instance interface signals. mbind builds an internal representation of the whole system based on an analysis of this specification and of the graph description files associated with each instantiated module. At this stage, the only information used from these files is the list of signals attached to each module.

Signal interconnections are represented with entities called nets, which ensure the propagation of the status and possible value of signals as they are determined. Fig.9 below shows the result for COMCTL with circles to symbolize the nets :

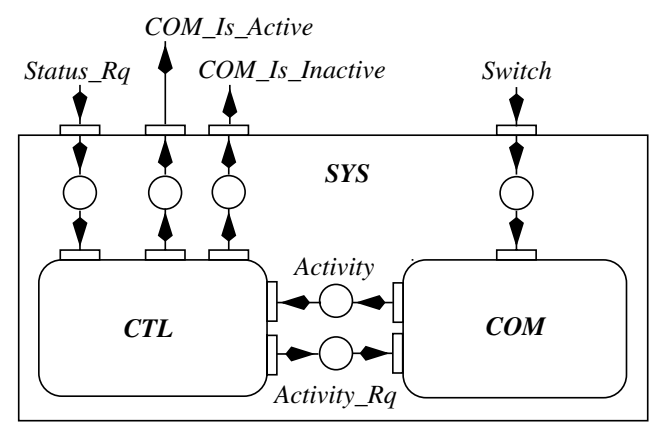

Fig. 9. Cronos global representation of ComCtr

The figure shows three kinds of nets : input nets which link a system input to an instance input, output nets which link an instance output to a system output, and internal nets which link an instance output to an instance input. They all represent an information flow between some producer and some consumer to which mbind associates an evaluation node performing the necessary propagation operations.

mbind then uses for each module the dependencies between its inputs/outputs and its partial evaluations to merge the evaluation graphs of all the instances via the net evaluation nodes. Fig.10 shows the result of this operation for the COMCTL example, also indicating that the mgen compression produced a graph with only one evaluation node.

As mgen, mbind finally performs a graph compression, generates a description file of the result and produces the corresponding code. 


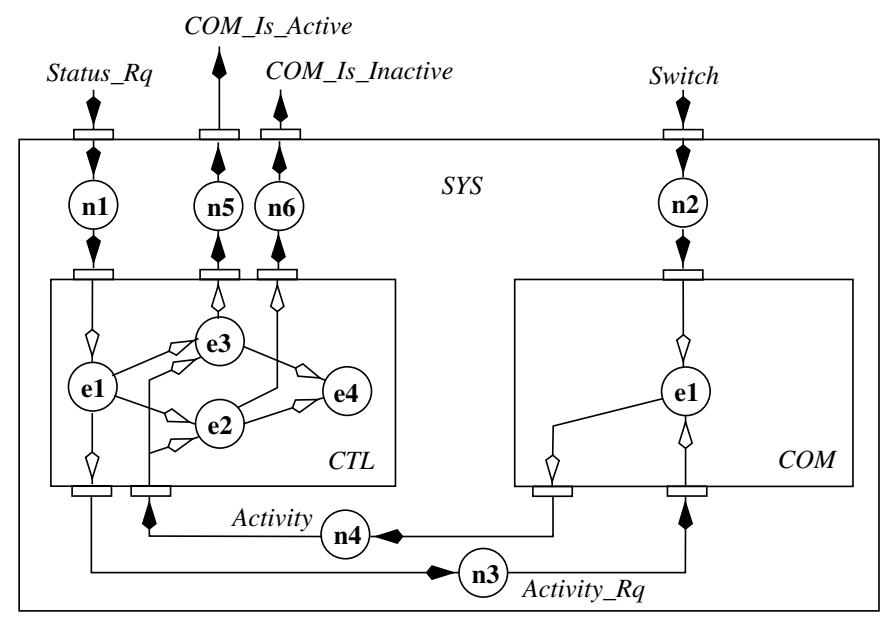

Fig. 10. Cronos graph integration for ComCTL

As already mentioned in 5.1, such a binding operation is only possible if the overall circuit is acyclic. To ensure this, mbind checks that the system evaluation graph is acyclic and indicates an error if it fails. In such cases, the cycles are exhibited in terms of signal dependencies and their resolution is left to the user.

The following sections describe the overall architecture of the generated code to provide a more concrete view of the process. The chosen target language is Ada because of its numerous good properties for the real-time domain and since its integration with other classical languages is easy anyway.

\subsection{Module Code Architecture}

From any EsTEREL module, mgen produces an Ada package which enables the exploitation of the corresponding circuit through a simple interface.

This interface includes one accessor (Set_I) for each input signal I, one accessor $(0)$ for each output signal 0 , one accessor for each partial evaluation node and four module-level entry points (Initialize, Reset, Run and Clear).

Initialize performs some internal initializations and shall be called once before anything else. Clear sets to Absent the status of all the module inputs and shall be called at least once before the beginning of any reaction. Reset sets the circuit registers to their initial value and shall be called at least once before the first reaction. Run triggers a one step complete reaction by calling all the evaluation procedures in topological order.

For any reaction in the context of which an input I should be considered present, Set_I shall be called before all the evaluation procedures accessing its status. The emission status of any output 0 can be retrieved via the corresponding accessor, which should only be called once it is sure that 0 is determined. 
Fig.[1] below shows the specification obtained for CTL :

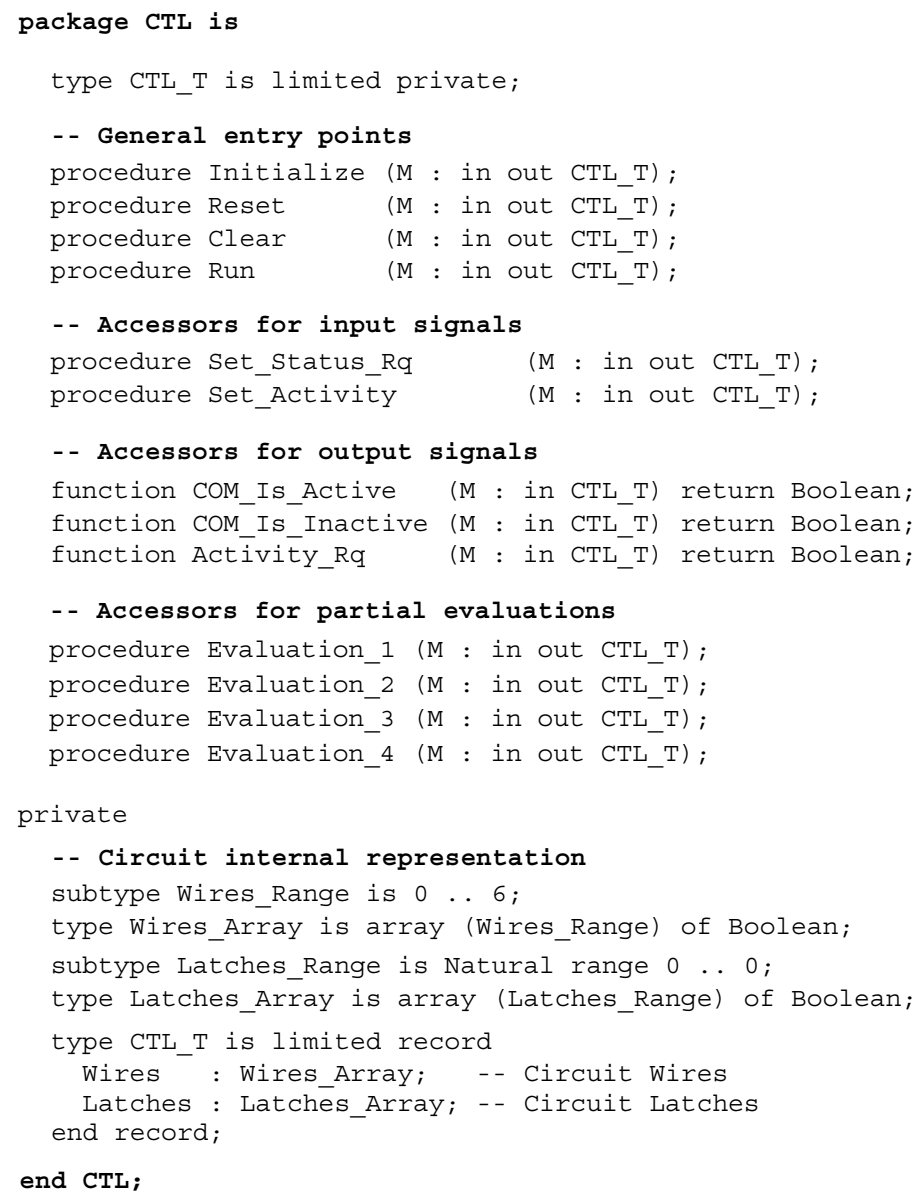

Fig. 11. Cronos Ada specification for the CTL module

The abstraction of the module circuit is provided through a private record type which contains all the circuit components and offers the possibility to safely instantiate the same module several times when necessary.

The first possible use of such a package is the instantiation of the corresponding module as an independent system. After a simple variable declaration with the provided type, the instance has to be initialized with Initialize and Reset. A sequence of reactions can then be triggered, each as a set of calls to Clear, to Set for every input to be considered present, and to Run to process the complete circuit evaluation. After every reaction the status of any output can be retrieved with the provided accessors. 
Thus, mgen does not only allow the separate compilation of a module to be integrated into an outer pure parallel system, but also provides an Ada code generation alternative for any ESTEREL specification. Section 8 provides some experiment results intended to evaluate its efficiency.

Fig. 12 below provides an overview of the package body for CTL :

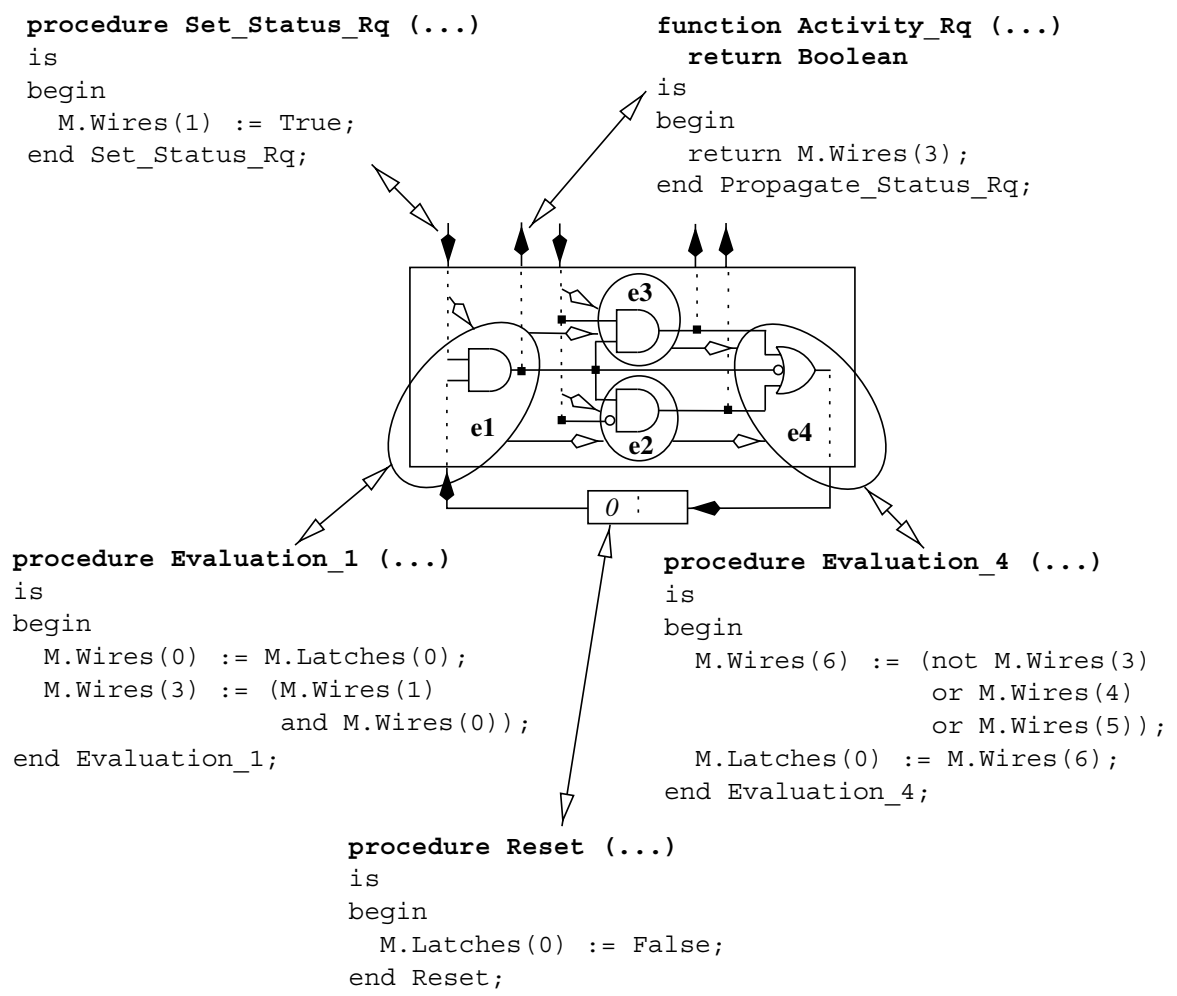

Fig. 12. Cronos evaluation splitting for the CTL module

In case instances of the module are integrated into a parallel composition, it is the responsibility of the code produced by mbind for this composition to use the services offered by the package in a consistent way.

\subsection{System Code Architecture}

mbind generates an Ada package which offers exactly the same kind of interface as the one provided by mgen for individual modules. The very detail of the system record is not important, but it is interesting to notice that it contains a set of fields for the instances from which the system is built and a set of variables for the status/value of the system interface signals. 
Initialize and Reset are propagated to each instance, as well as Clear which additionally resets the status of the system interface signals. Fig. 13 shows the sequence of reactions of Fig. 3 with the simplified code for the initialization of the system and using Run for the logical instants 3 and 4 .

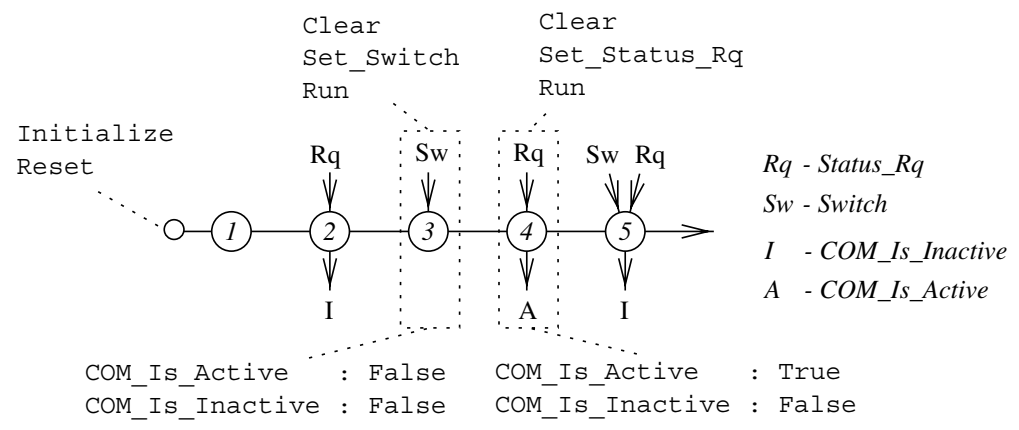

Fig. 13. Example sequence of reactions for COMCTL

As previously described, the system evaluation nodes are a combination of net evaluation nodes and integrated instance nodes, possibly grouped by a compression algorithm. Evaluation of instance nodes are translated into calls to the corresponding evaluation procedure and Fig. 14 shows the translation for internal nets achieving the signal propagations between modules.

Each system node is supported by an evaluation procedure and Run contains a topologically ordered sequence of calls to these procedures which ensures the respect of all the precedence constraints.
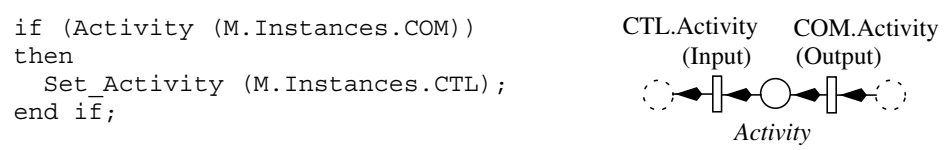

Fig. 14. An example of Cronos net evaluation node

The processing for input or output nets is similar, but uses the internal record variables storing the status of the system interface signals. Handling valued signals and signals with multiple producers and/or consumers, allowed by ESTEREL, requires a little more processing, both at the mgen and mbind levels. Such processing is available in CRONOs but does not change any fundamental aspect of our solution regarding the separate compilation issue, so we will not provide information about it. 


\section{Integration within the Esterel Toolset}

Fig.15 provides a user-level overview of the whole separate compilation process for COMCTL. The . ads and . adb files are respectively Ada package specifications and Ada package bodies. The .dep files are the graph description files produced by mgen from its analysis of each module (dep stands for dependencies).

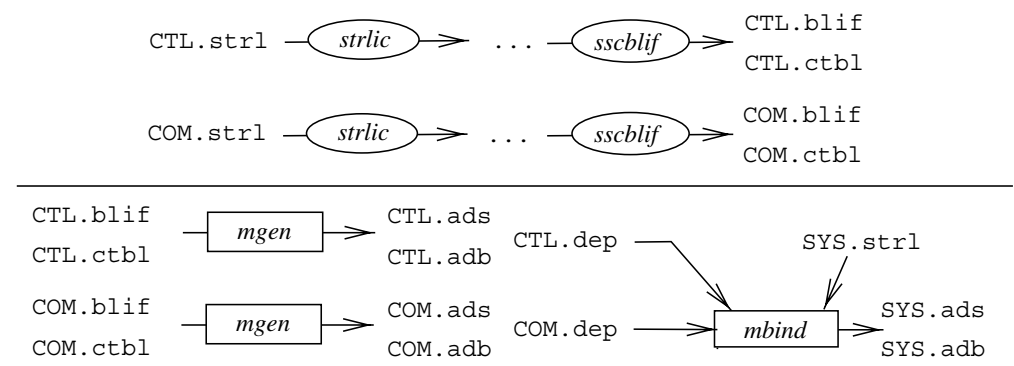

Fig. 15. Esterel/Cronos integration for separate compilation

This process clearly delays the modules assembly step and still uses the vast majority of the existing tools, so CRONOs will benefit from all their possible future enhancements.

\section{Major Advantages}

Cronos both provides an Ada alternative to the existing $\mathrm{C}$ code production from EsTEREL and separate compilation framework for specifications with pure parallelism at the root level. CRONOS also supports hierarchically organized systems, where the highest level modules are themselves written as pure parallel module instantiations, and so possibly recursively.

The first benefits are classical regarding separate compilation in general : the code for an unchanged module can be used directly in different contexts and does not have to be generated again when a system is rebuilt. The operations applied to a global representation of a system to rebuild it after a local modification are minimized, and in case of a "from scratch" build, most of the translation steps are only applied to one module at a time. The latter point is very strong because algorithms such as the causality analysis mentioned in 4.3 are sometimes too time and memory demanding for a large system as a whole. In such cases, Cronos offers an alternative to succeed in obtaining a final result.

Moreover, since CRONOS uses the blif representation of each module as a starting point, it is possible to build a system from an optimized version of each of its components. This may enable the production of a system of acceptable size and performance when a global optimization is not possible. 
Finally, the requirements about the root-level parallelism and the global graph acyclicity seem to be reasonable in the case of carefully designed modular applications. Several large projects have actually been developed this way by Dassault-Aviation and respecting these constraints has never been considered as a true obstacle. Actually, some rare global cycles appeared, but always corresponded to design flaws and have been easily solved thanks to their expression in terms of interface signal dependencies without intra-module paths.

\section{Experiments and Results}

Two significant prototype applications have been developed in the context of our studies. The first one handles a small part of the specification for the computing system of one aircraft model. The second one is a model of fuel management used in a more general simulation environment. They will be denoted by ACS for the Aircraft Computing Subsystem and FMS for the Fuel Management Simulation. Table 1 provides general information about their source :

Table 1. Source characteristics for ACS and FMS

\begin{tabular}{lccc}
\hline System & Lines & Modules & Instances \\
\hline ACS & 4500 & 14 & 14 \\
FMS & 4560 & 7 & 14 \\
\hline
\end{tabular}

"Lines" indicates the number of Esterel lines involved, "Modules" the number of separately compiled modules, and "Instances" the number of instances of such modules in the system. The ESTEREL code has been generated from SYNCCharts [7] graphical specifications.

The next table is intended to compare the efficiency of the generated code for four different compilation processes. The first one (further denoted by "CMono") is the standard EsTEREL scheme producing monolithic C code without intermediary blif optimizations. The second one ("Ada-Mono") produces monolithic Ada code via mgen without prior blif optimization by considering the main EsTEREL source itself as a single module. The third one ("AdaModular") is a CRONOS separate compilation without blif optimization. The last one ("Ada-Opt-Modular") is a CRONOS separate compilation after some optimizations performed on the blif representation of each module. In every case, the system has first been submitted to a simulation test to ensure that its behavior corresponds to a validation reference (what output sequence from a set of reactions with predefined inputs). Then a simple main procedure has been written for the target language to trigger 10000 reactions. Executables have been generated with the same class of compiler/options and run several times on the same machine (RS6000/41T-PowerPC-AIX 4.2.1) with no other user logged in. 
Table 2 below provides the mean per-reaction execution times observed :

Table 2. Mean reaction execution times for ACS and FMS

\begin{tabular}{lcccc}
\hline System & C-Mono & Ada-Mono & Ada-Modular & Ada-Opt-Modular \\
\hline ACS & $1.01 \mathrm{~ms}$ & $0.93 \mathrm{~ms}$ & $1.32 \mathrm{~ms}$ & $0.41 \mathrm{~ms}$ \\
FMS & $1.58 \mathrm{~ms}$ & $1.36 \mathrm{~ms}$ & $1.28 \mathrm{~ms}$ & $0.35 \mathrm{~ms}$ \\
\hline
\end{tabular}

The comparison of the "C-Mono" and "Ada-Mono" columns shows that the Ada code generation is efficient by itself since it yields slightly better results starting from the same ssc representation of the entire system.

Comparing "Ada-Mono" and "Ada-Modular" requires a careful look at the statistics from Table 1]: ACS instantiates each module only once whereas FMS instantiates some modules several times. In the monolithic case, the instantiation of the parallel modules is performed by the ESTEREL compiler which basically expands the code for each instance. In the modular case, these instantiations are translated into variable declarations, but the code for different instances of the same module is not duplicated. Some portions of code are then shared in FMS and not in ACS. The execution time increase between Mono and Modular for ACS is due to the addition of the net evaluation nodes for inter-module communications. Such nodes are naturally also present for FMS, but it seems that the code sharing balances the overhead, probably because it results in a better cache utilization.

Finally, the last column clearly demonstrates the interest of the possibility to bind optimized modules, especially when a global optimization is not tractable (which was indeed the case for these applications on our machine).

\section{Conclusion and Work in Progress}

Cronos both provides an efficient Ada alternative to the existing $\mathrm{C}$ code production from ESTEREL and separate code generation schemes for modular specifications. This greatly increases the maximum size of systems for which runnable code can be obtained and provides a code production process more suitable to large industrial applications. This enables to take advantage of the ESTEREL determinism and formal verification features in the context of large avionics software developments such as the ones Dassault-Aviation leads.

Much related work is still in progress in the company. The most important activities are the development of an integrated graphical environment, a $\mathrm{PhD}$ thesis about the distributed execution of separately compiled modules, and studies about a model of asynchronous interconnection of synchronous systems.

All of these projects are conducted in cooperation with the ESTEREL team of École des Mines de Paris and Centre de Mathématiques Appliquées at Sophia Antipolis, constantly improving the EsTEREL technology. 


\section{References}

[1] Berry, G., The Foundations of Esterel, École des Mines de Paris (1998) ${ }^{1}$

[2] Berry, G., The constructive semantics of pure Esterel - Draft V2.0, École des Mines de Paris (May, 1996) ${ }^{1}$

[3] Berry, G., The Esterel v5 Language Primer, École des Mines de Paris (1997) ${ }^{1}$

[4] Berry, G., Bouali, A., Fornari, X., Ledinot, E., Nassor, E., and De Simone, R., Esterel : A Formal Method Applied to Avionic Software Development, To appear in Journal of Science of Computer Programming (1998)

[5] Hainque, O., Pautet, L., Le Biannic, Y., and Nassor, E., Using Esterel for Avionics Software Development : Motivation, Experiments and Work in progress, In Proc. of the 1998 Workshop on Programming Languages for Real-Time Industrial Applications, (December, 1998), 9-18

[6] E.M. Sentovic, K.J. Singh and L. Lavagno, C. Moon , R. Murgai,A. Saldanha, H. Savoj, P.R. Stephan, R.K. Brayton, andA.L. Sangiovanni-Vincentelli SIS : A System for Sequential Circuit Synthesis, University of California Berkeley : UCB/ERL M92/41 (1992)

[7] C. André, M. Bourdellès and S. Dissoubray SyncCharts/Esterel : Un environnement graphique pour la spécification et la programmation d'applications réactives complexes, In Revue du Génie Logiciel (46) (1997)

[8] G. Berry, T.R. Shiple, H. Touati, Constructive Analysis of Cyclic Circuits, (March, 1996), ED-TC 328-333

[9] H.A. Toma, Analyse constructive et optimisation séquentielle des circuits générés à partir du langage synchrone réactif Esterel, PhD thesis, École des Mines de Paris (September, 1997) ${ }^{1}$

[10] Amagbegnon, T.P., Forme canonique arborescente des horloges de SignAL, PhD thesis, Université de Rennes I (December, 1995)

[11] Beneviste, A., Le Guernic, P., and Jacquemot, C. Synchronous programming with events and relations : the SignAL language and its semantics, In Science of Computer Programming, v.16, (1991)

[12] Halbwachs, N., Caspi, P., Raymond, P., and Pilaud, D. The Synchronous dataflow programming language Lustre, Proceedings of the IEEE, 79(9):1305-1320, (September, 1991)

[13] André, C., Boulanger, F., Péraldi, M.-A., Rigault, J.P., and Vidal-Naquet, G. Objects and Synchronous Programming, In European Journal on Automated Systems, v.31(3), 417-432, Hermes (1997)

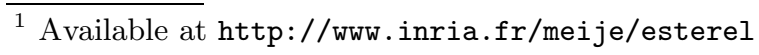

